\title{
Qualidade das notificações de violências interpessoal e autoprovocada no Estado do Rio de Janeiro, Brasil, 2009-2016
}

\author{
Quality of the notifications of interpersonal and \\ self-inflicted violence in the state of Rio de Janeiro, Brazil, 2009-2016
}

\author{
Vania Reis Girianellii*, Aldo Pacheco Ferreira ${ }^{1}$, Marcos Besserman Vianna ${ }^{1}$, \\ Nair Teles ${ }^{1}$, Regina Maria de Carvalho Erthal ${ }^{1}$, Maria Helena Barros de Oliveira ${ }^{1}$
}

\begin{abstract}
Resumo
Introdução: Os dados dos Sistemas de Informação em Saúde são muito utilizados para a produção do conhecimento. Para a sua efetiva utilização, no entanto, é essencial assegurar que sejam válidos e confiáveis. Objetivo: Avaliar a qualidade das notificações de violências no estado do Rio de Janeiro, visando contribuir para o aprimoramento e melhoria da informação. Método: Estudo ecológico sobre a consistência das notificações de violência registradas no Sistema de Informação de Agravos de Notificação do Estado do Rio de Janeiro, entre 2009 a 2016. A tendência temporal das proporções de inconsistências, para cada grupo de campos relacionados, foi avaliada por regressão linear simples e respectiva significância estatística $(p \leq 0,05)$. Resultados: No período foram notificados 103.841 casos de violência, mas $31,7 \%$ não foram identificados se interpessoal ou autoprovocada. As principais inconsistências foram: incongruência entre violência psicológica e meio da agressão, sexo e identidade de gênero, tipo de violência e idade da vítima, violência autoprovocada e características do agressor e tipo de violência; a maioria com estabilidade no período ( $p>0,05)$. Conclusão: Os resultados evidenciam inconsistências expressivas nas notificações de violência que comprometem a utilização da informação.

Palavras-chave: sistema de informação em saúde; doenças e agravos de notificação compulsória; violência doméstica; tentativa de suicídio; direitos humanos.

Background: Data from Health Information Systems are widely used for the production of knowledge. For their effective use, however, it is essential to ensure that they are valid and reliable. Objective: Evaluate the quality of reports of violence in the state of Rio de Janeiro aiming to contribute to the improvement of information. Method: Ecological study on the consistency of notifications of violence recorded to the Information System on Diseases of Compulsory Declaration in the state of Rio de Janeiro from 2009 to 2016. The temporal trend of the proportions of inconsistencies, for each group of related fields, was evaluated by simple linear regression and its statistical significance $(p \leq 0.05)$. Results: During the study period, 103,841 cases of violence were reported, but in $31.7 \%$ of them it was not possible to identify whether they were interpersonal or self-inflicted. The main inconsistencies were incongruence between psychological violence and aggression, gender identity and sex, type of violence and age of the victim, self-inflicted violence and characteristics of the aggressor and type of violence; most of them presented stability in the period ( $p>0.05$ ). Conclusion: The results show significant inconsistencies in the reports of violence that compromise the use of this information.
\end{abstract}

\begin{abstract} Keywords: health information systems; disease notification; domestic violence; attempted suicide; human rights.

'Departamento de Direitos Humanos, Saúde e Diversidade Cultural (DIHS), Escola Nacional de Saúde Pública Sérgio Arouca (ENSP), Fundação Oswaldo Cruz (FIOCRUZ) - Rio de Janeiro (RJ), Brasil.

Trabalho realizado na Escola Nacional de Saúde Pública Sérgio Arouca (ENSP) - Rio de Janeiro (RJ), Brasil.

*Endereço para correspondência: Vania Reis Girianelli - Departamento de Direitos Humanos, Saúde e Diversidade Cultural (DIHS), Escola Nacional de Saúde Pública Sérgio Arouca (ENSP), Fundação Oswaldo Cruz (FIOCRUZ), Avenida Brasil, 4036, Sala 905 - Manguinhos - CEP: 521040-361 - Rio de Janeiro (RJ), Brasil - Email: vaniagirianelli@yahoo.com.br

Fonte de financiamento: nenhuma.

Conflito de interesses: nada a declarar.
\end{abstract}

Este é um artigo publicado em acesso aberto (Open Access) sob a licença Creative Commons Attribution, que permite uso, distribuição e reprodução em qualquer meio, sem restrições desde que o trabalho original seja corretamente citado. 


\section{INTRODUÇÃO}

O Sistema de Informação em Saúde (SIS) do Brasil é constituído por vários subsistemas que contemplam dados referentes a mortalidade, nascimento, morbidade e assistência à saúde, que são fundamentais para formulação e avaliação das políticas, planos e programas de saúde, subsidiando o processo de tomada de decisões. Os dados dos SIS são disponibilizados no site do Departamento de Informática do Sistema Único de Saúde (DataSUS) do Ministério da Saúde (MS), e também no site institucional de alguns estados e municípios ${ }^{1}$.

O Sistema de Informação de Agravos de Notificação (Sinan) é um dos subsistemas do SIS que foi implantado na década de 1990. Este sistema é alimentado, principalmente, com dados que constam da ficha de notificação individual de doenças e agravos, incluídos na lista nacional de notificação compulsória (LNNC) ${ }^{2}$. Esta lista é composta de doenças e agravos relevantes tendo em vista a magnitude, potencial de disseminação, transcendência, vulnerabilidade, compromissos internacionais e regulamento sanitário internacional. Os estados e municípios também podem acrescentar, na sua região, outras questões de saúde pública relacionadas ao contexto local ${ }^{3}$.

As violências autoprovocadas e interpessoais só passaram a integrar a LNNC em 20114. A notificação de violência interpessoal abrange doméstica/intrafamiliar; sexual; tráfico de pessoas; trabalho escravo; trabalho infantil; tortura; intervenção legal; violências homofóbicas; e também violência extrafamiliar/comunitária, mas restrita a populações vulneráveis (crianças, adolescentes, mulheres, idosos, deficientes, indígenas, lésbicas, gays, bissexuais, travestis e transexuais) ${ }^{5}$. Posteriormente, as notificações de violência sexual e de tentativa de suicídio passaram a ser de notificação imediata no nível municipal ${ }^{6}$, visando garantir o acesso às medidas de prevenção e assistência em tempo oportuno. Na penúltima versão da ficha de notificação ${ }^{7}$ foram incluídos os campos "orientação sexual", "identidade de gênero" e "motivo da violência"; mas estes dados, por enquanto, só estão disponíveis no site do estado do Rio de Janeiro.

A notificação de violências, no entanto, já havia sido implantada anteriormente, como previsto nas legislações (Estatuto da Criança e do Adolescente - ECA ${ }^{8}$, Estatuto do Idoso $^{9}$, violência contra à mulher ${ }^{10}$ e prevenção de suicídio ${ }^{11}$ ), mas era restrito a serviços de referência (Doenças Sexualmente Transmissíveis/Síndrome da Imunodeficiência Adquirida DST/Aids, ambulatórios especializados, maternidades, entre outros) definidos pelas secretarias municipais e estaduais de saúde. Cabe ressaltar que em 2009 a notificação de violências foi inserida no Sinan, mas de forma gradual conforme a adesão dos estados e municípios.
Os dados dos SIS são muito utilizados para a produção do conhecimento. Para a sua efetiva utilização, no entanto, é essencial assegurar que sejam válidos e confiáveis. A ficha de notificação de violências, além de ser a mais recente a ser implantada, é a mais complexa do sistema. Nesta ficha foram unificadas diversas fichas de notificação existentes anteriormente (tentativa de suicídio, maus tratos da criança e adolescente, maus tratos do idoso e violência contra mulher), e também contém campos que envolvem conceitos atualmente discutidos na sociedade como identidade de gênero, comportamento sexual e sexismo; cujos significados podem ainda não ser de domínio dos profissionais que atendem à demanda, e consequentemente levar a erros de classificação.

O presente estudo tem como objetivo avaliar a qualidade das notificações de violência no estado do Rio de Janeiro, visando contribuir para o aprimoramento e melhoria da informação.

\section{MÉTODO}

Trata-se de estudo ecológico sobre a qualidade das notificações de violências no estado do Rio de Janeiro no período de 2009 a 2016. Os dados foram obtidos do Sinan, disponibilizado com acesso livre no site da Secretaria de Estado de Saúde do Rio de Janeiro. Foram elegíveis para o estudo apenas as violências notificadas nos municípios do estado.

A dimensão da qualidade analisada foi apenas a consistência interna ${ }^{12,13}$, que aprecia a existência de coerência entre os campos. Esta avaliação baseou-se na recomendação do instrutivo de preenchimento de notificação de violência preconizado pelo Ministério da Saúde ${ }^{14}$. Os campos avaliados foram: idade, sexo, gestação, escolaridade, situação conjugal/estado civil, orientação sexual, identidade de gênero, lesão autoprovocada, motivação da violência, tipo de violência, meio de agressão, tipo de violência sexual, procedimento realizado, sexo do provável agressor, número de agressores envolvidos e vínculo com a pessoa atendida.

A investigação consistiu em identificar a existência de inconsistência entre os campos relacionados, sendo classificada em:

a) Não conformidade - preenchimento inadequado, mas que não compromete totalmente a compreensão ou análise dos dados. Situação em que a classificação correta de um campo seria "Não se Aplica", mas é classificado como "ignorado", "não", ou o campo não é preenchido.

b) Incongruência: classificação de dois campos distintos de forma que não sejam simultaneamente verdadeiros, comprometendo a compreensão e consequentemente a análise de dados. Situação, por exemplo, em que uma vítima adulta tem a violência sofrida classificada como pornografia infantil, ou seja, um dos campos foi preenchido incorretamente. 
O percentual de inconsistência foi calculado considerando no numerador a quantidade fichas com inconsistência para determinadas categorias dos campos relacionados, conforme exemplificado anteriormente, e no denominador apenas o quantitativo de fichas de notificação que continham as categorias dos campos sob análise. As inconsistências relativas a não conformidade foram classificadas como baixa quando menor de $10 \%$, moderada entre $10 \%$ a $30 \%$ e alta quando maior que $30 \%$. Já as incongruências foram classificadas como baixa quando menor que $0,5 \%$, moderada entre $0,5 \%$ a $10 \%$ e alta quando maior que $10 \%$.

Calculou-se o percentual de inconsistência anual e a mediana para o período, para cada grupo de campos relacionados. A tendência temporal foi avaliada por regressão linear simples, tendo como variável independente o ano de notificação e como variável dependente o percentual de inconsistência. $\mathrm{O}$ aumento ou declínio da proporção de inconsistência no período foi avaliado com base no coeficiente de regressão $(\beta)$ e respectiva significância estatística $(\mathrm{p} \leq 0,05)$. As análises foram realizadas utilizando o programa estatístico $\mathrm{R}$.

$\mathrm{O}$ estudo foi realizado utilizando dados secundários, de acesso público, sem identificação individual das informações. Assim, consoante às recomendações da Resolução do Conselho Nacional de Saúde (CNS) no 466, de 12 de dezembro de 2012, foram respeitados os princípios éticos de pesquisas que envolvem seres humanos.

\section{RESULTADOS}

No período avaliado foram notificados 103.841 casos de violência pelos municípios do estado do Rio de Janeiro, sendo $62,5 \%$ violência interpessoal e 5,7\% autoprovocada, os demais foram classificados como ignorado ou não preenchidos $(31,7 \%)$.

A incongruência entre a idade e outros campos foi baixa $(<0,2 \%)$ em todos os anos (Tabela 1). Já a inconsistência relacionada a não conformidade foi moderada entre "idade" $\mathrm{e}$ "pornografia infantil" (Mediana $=29,7 \%$ ) e baixa entre idade e situação conjugal (Mediana $=5,2 \%$ ). O campo "trabalho infantil" não tem a opção de categorização "Não se Aplica" e não teve nenhum registro classificado como "Ignorado". Análise complementar foi realizada considerando a idade de 14 anos ou mais em relação ao trabalho infantil, devido a possibilidade de trabalho como aprendiz ${ }^{15}$; e também para 18 anos ou mais no que se refere à pornografia infantil, tendo em vista a classificação de maioridade no $\mathrm{ECA}^{8}$. Os resultados, no entanto, foram similares (dados não mostrados).

A Tabela 2 apresenta as inconsistências relacionadas às notificações de violência autoprovocada. A proporção de incongruência foi moderada ou alta para maioria dos campos avaliados $(>0,5 \%)$, com aumento estatisticamente significativo no período para os campos sexo do agressor $(\beta=0,34 ; \mathrm{p}=0,004)$ e número de agressores envolvidos $(\beta=0,26 ; \mathrm{p}=0,020)$. A mediana das não conformidades foi moderada, variando de $11,2 \%$ para estupro a 14,3\% para exploração sexual, com redução estatisticamente significativa no período para a maioria dos campos avaliados $(\mathrm{p}<0,05)$. O campo atentado violento ao pudor não consta na nova versão da ficha de notificação, tendo sido incorporado ao estupro, de forma a manter consonância com a nova legislação ${ }^{16}$. Este campo, portanto, foi avaliado do período de 2009 a 2014, tendo apresentado baixa incongruência (Mediana $=0,2 \%$ ) e moderada não conformidade (Mediana $=17,5 \%$ ); ambos com tendência estável no período ( $\beta=-1,60, \mathrm{p}=0,504$ e $\beta=-0,11, \mathrm{p}=0,453$; respectivamente).

Em relação à notificação de violência interpessoal foram notificados mais de um tipo por vítima (dados não mostrados), embora a recomendação seja registrar apenas o principal ${ }^{14}$. A inconsistência foi uniforme no período avaliado $(p>0,05)$.

A Tabela 3 apresenta as inconsistências relacionadas a violência interpessoal do tipo psicológica. A proporção de incongruências variou de moderada a alta (Mediana >0,5\%), exceto para os campos agressão por envenenamento e exploração sexual. Os campos agressão por força corporal ou espancamento $(\beta=0,30 ; p=0,001)$, agressão por enforcamento $(\beta=1,22 ; \mathrm{p}=0,033)$ e agressão por objeto contundente $(\beta=1,79 ; p=0,048)$ apresentaram aumento estatisticamente significativo da incongruência no período. A mediana da inconsistência por não conformidade variou de $16,6 \%$ (estupro) a $29,3 \%$ (exploração sexual), todos com declínio no período $(\mathrm{p}<0,05)$.

Dados sobre motivo da violência estão disponíveis apenas a partir de 2013. Neste período ocorreram 1.104 notificações de violência interpessoal por motivo de sexismo. Destas, 43 (3,9\%) eram vítimas do sexo masculino (dados não mostrados).

Dados sobre identidade de gênero estão disponíveis a partir de 2014. Neste período ocorreram 198 notificações de violência de travestis e transexuais (Tabela 4). Destas, 53,5\% correspondiam a mulheres transexuais, cujo o sexo biológico era feminino, $12,6 \%$ a homens transexuais com sexo biológico masculino, e duas notificações de travestis com sexo biológico feminino. A incongruência entre os campos identidade de gênero e sexo biológico foi muito alta $(62,7 \%)$.

Não houve inconsistência entre sexo biológico masculino e gestação, e entre os seguintes procedimentos: coleta de secreção vaginal, contracepção de emergência ou aborto previsto em lei (dados não mostrados). 


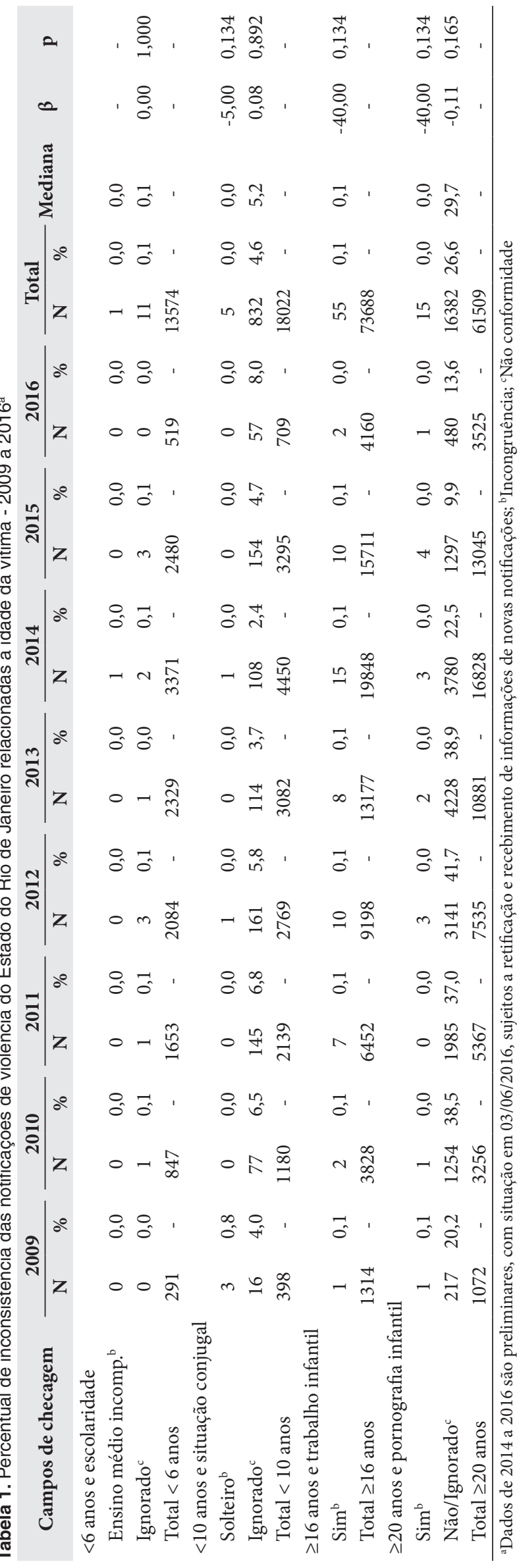




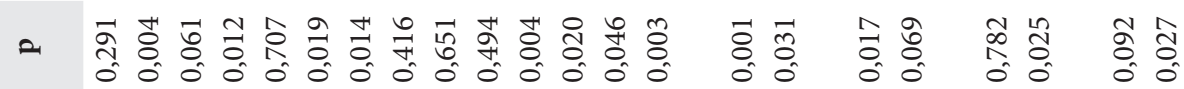

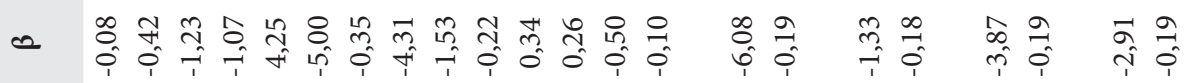

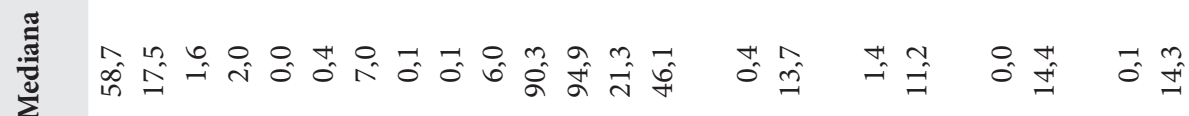

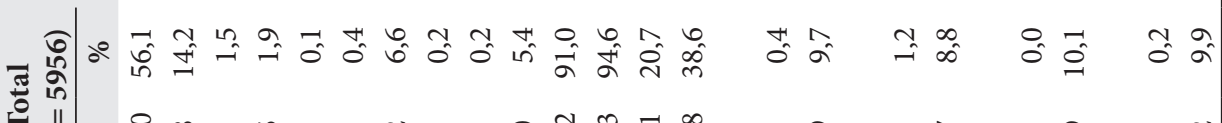

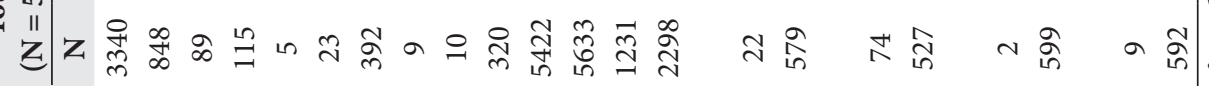

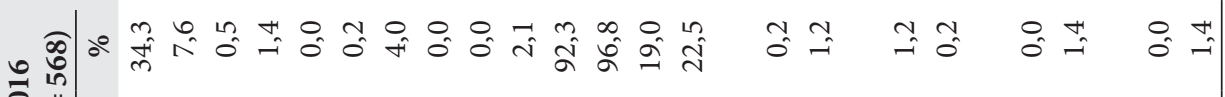

สై

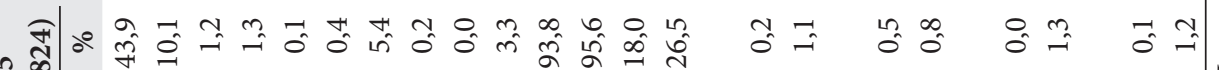

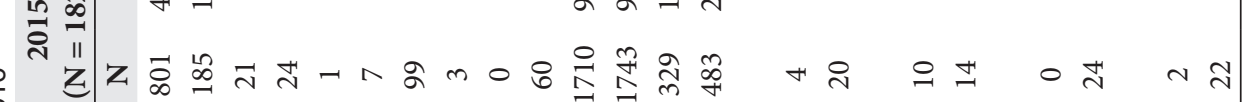

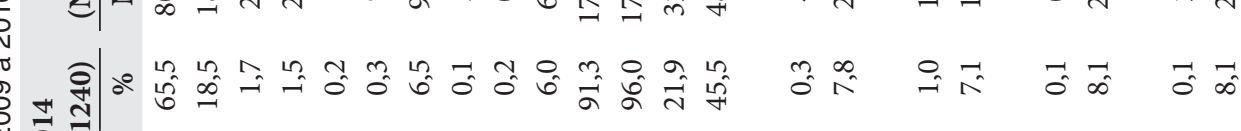

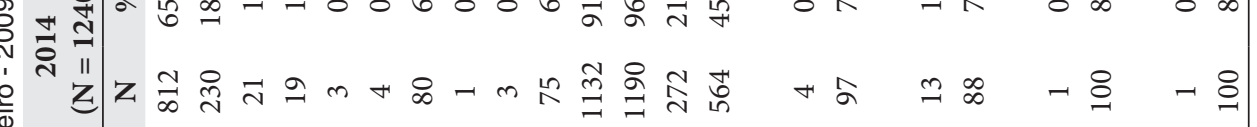

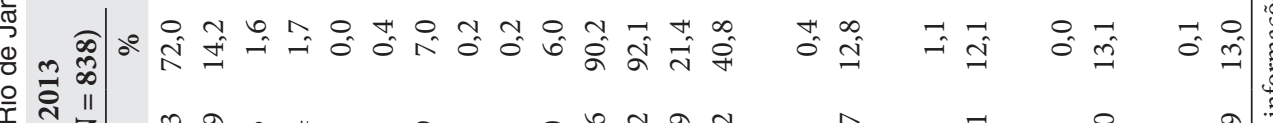

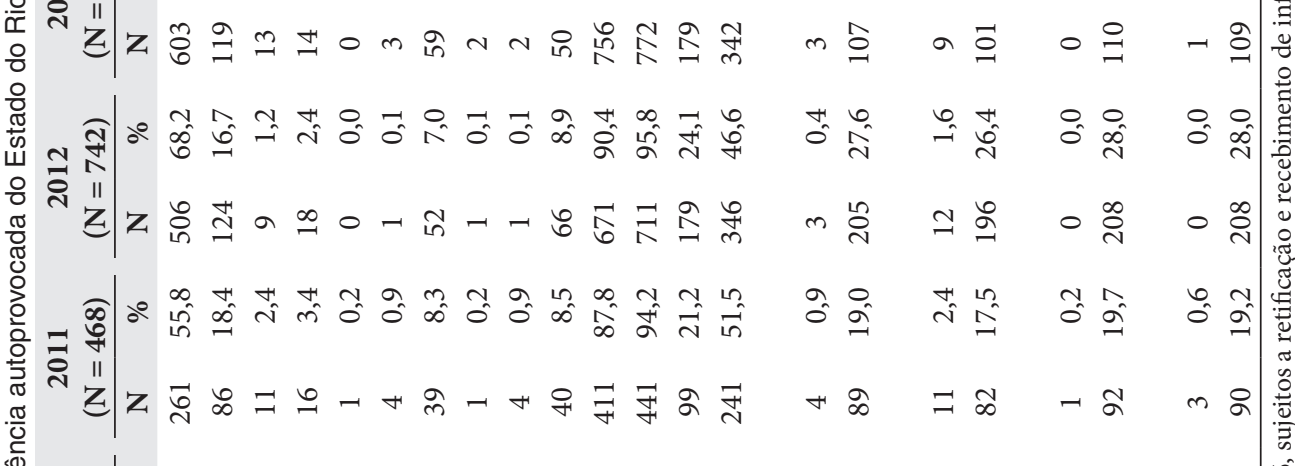

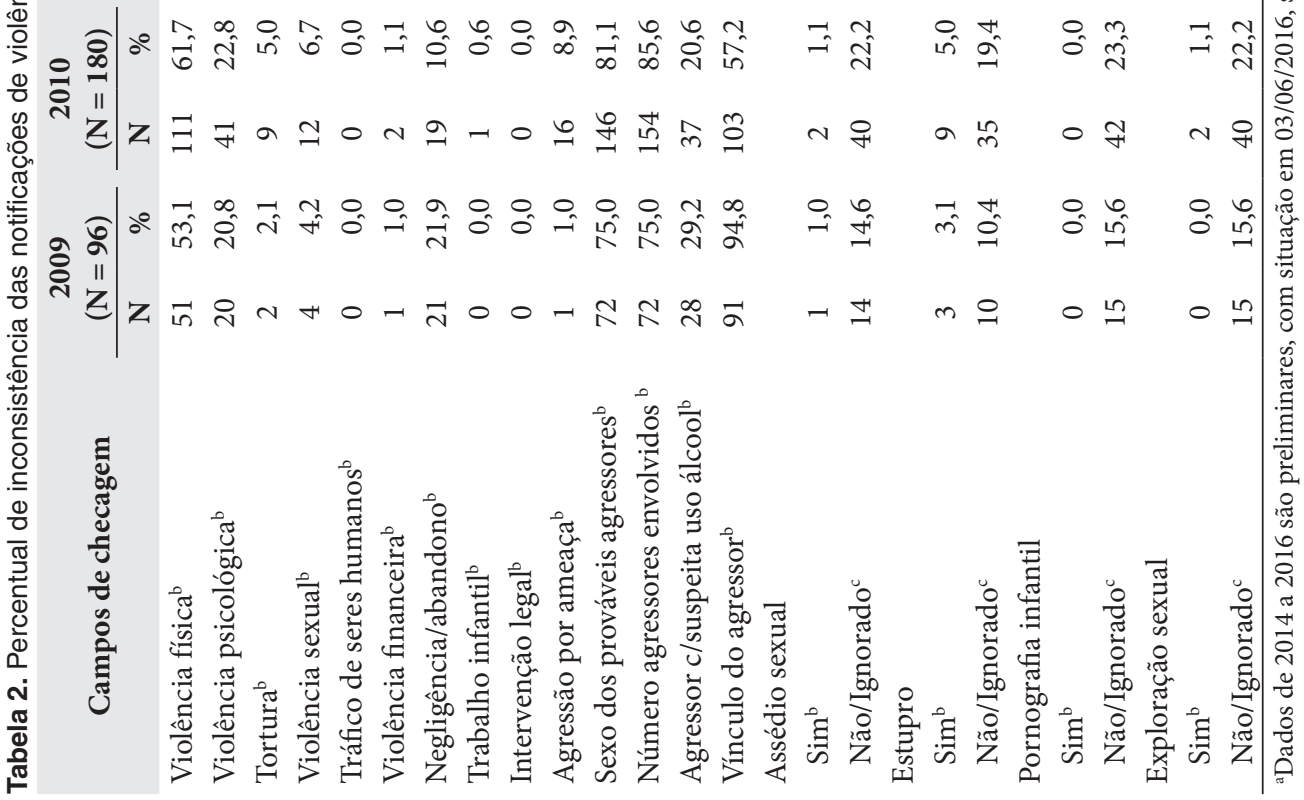




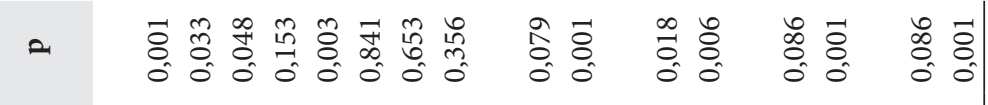

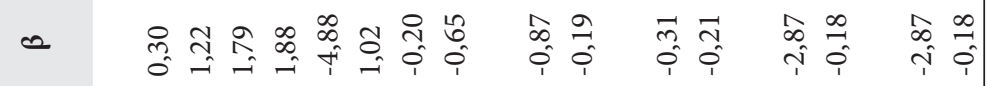

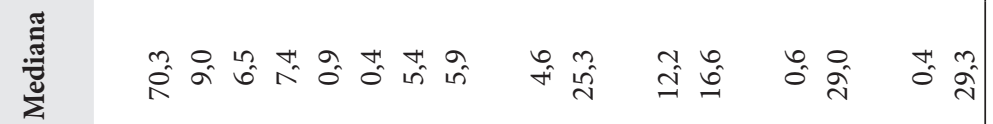

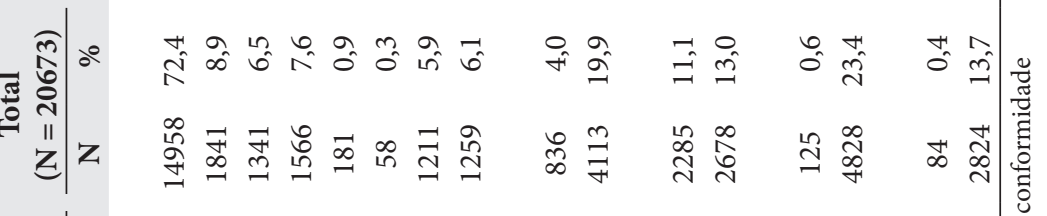

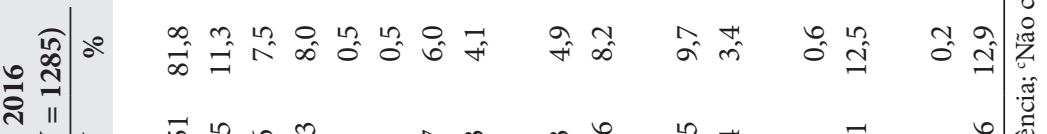

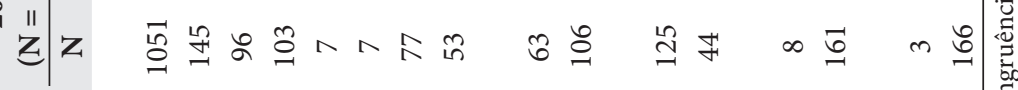

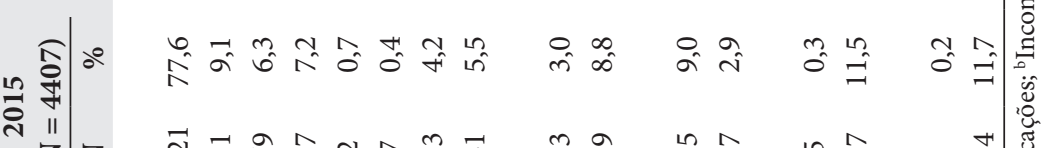

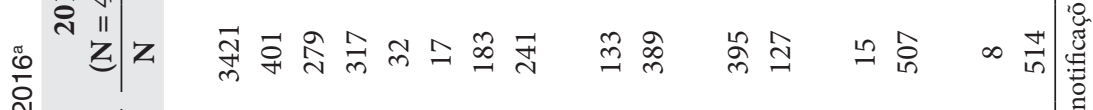

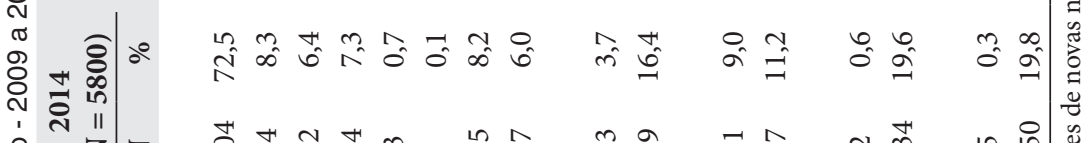

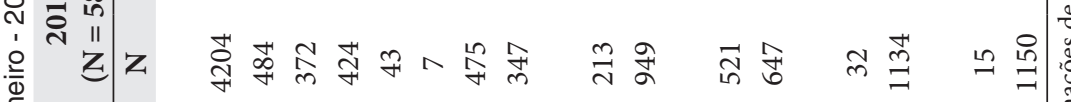

क्ष

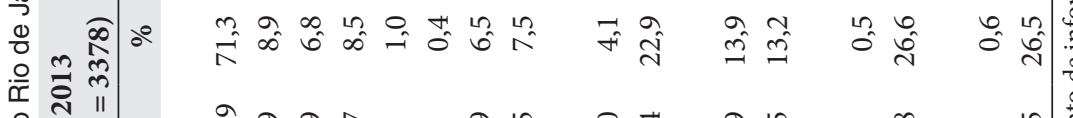

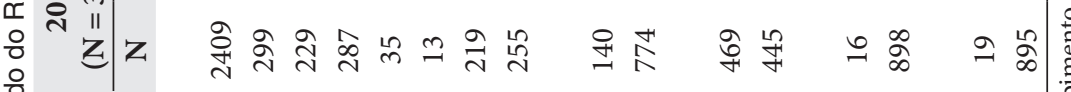

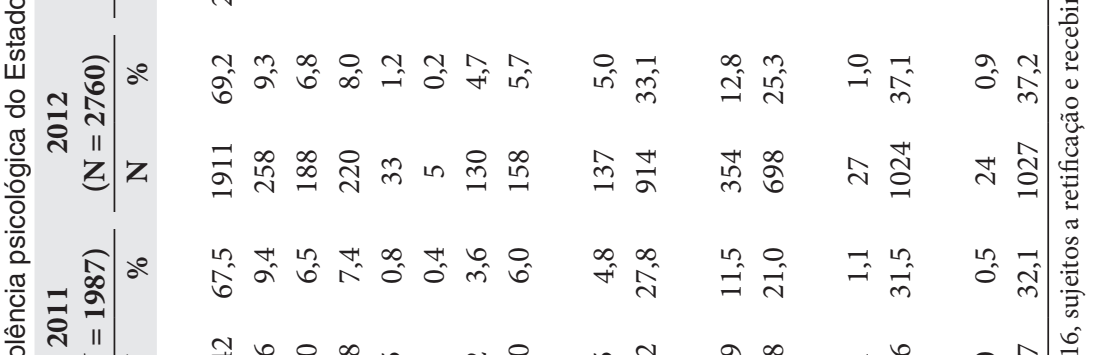

竞 $z$ z

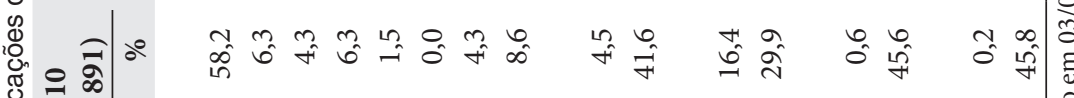

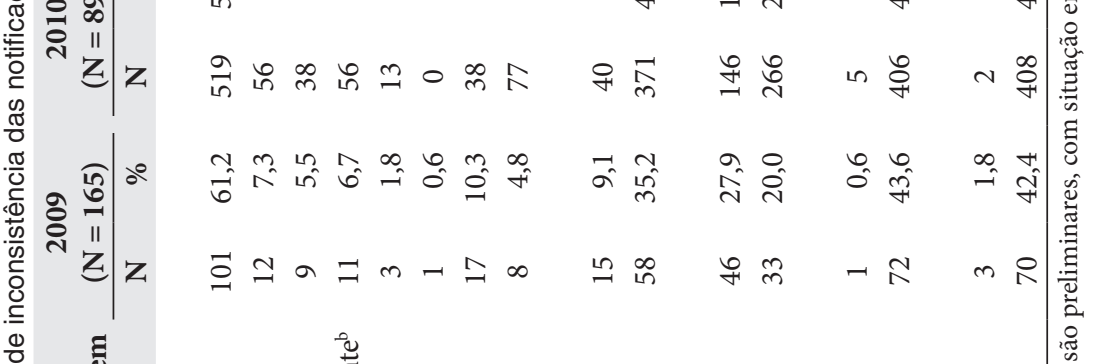

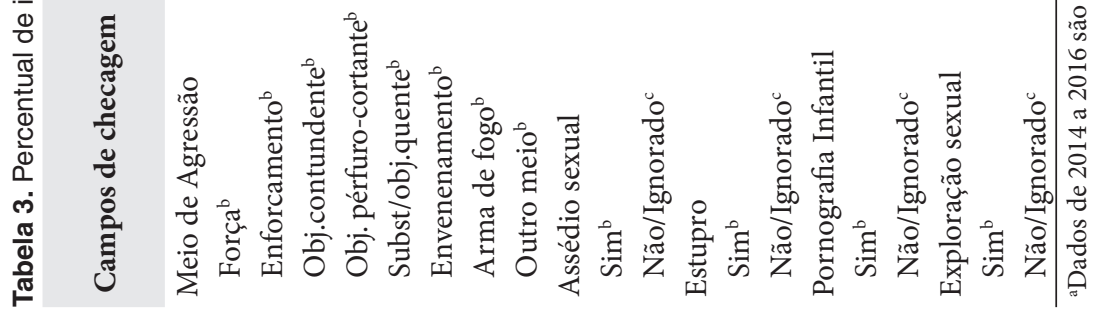


Tabela 4. Percentual de inconsistência das notificações de violência do Estado do Rio de Janeiro sofridas por travestis e transexuais por sexo biológico - 2014 a $2016^{a}$

\begin{tabular}{|c|c|c|c|c|c|c|c|c|}
\hline \multirow{2}{*}{ Campos de checagem } & \multicolumn{2}{|c|}{$2014(\mathrm{~N}=27)$} & \multicolumn{2}{|c|}{$2015(\mathrm{~N}=121)$} & \multicolumn{2}{|c|}{$2016(\mathrm{~N}=50)$} & \multicolumn{2}{|c|}{ Total $(\mathrm{N}=198)$} \\
\hline & $\mathbf{N}$ & $\%$ & $\mathbf{N}$ & $\%$ & $\mathbf{N}$ & $\%$ & $\mathbf{N}$ & $\%$ \\
\hline Sexo Feminino e Travesti & 0 & 0,0 & 2 & 1,7 & 0 & 0,0 & 2 & 1,0 \\
\hline Sexo Feminino e Mulher transexual & 16 & 59,3 & 65 & 53,7 & 25 & 50,0 & 106 & 53,5 \\
\hline Sexo Masculino e Homem transexual & 3 & 11,1 & 17 & 14,0 & 5 & 10,0 & 25 & 12,6 \\
\hline Total & 19 & 70,4 & 84 & 69,4 & 30 & 60,0 & 133 & 67,2 \\
\hline
\end{tabular}

a'Dados de 2014 a 2016 são preliminares, com situação em 03/06/2016, sujeitos a retificação e recebimento de informações de novas notificações

\section{DISCUSSÃO}

Os resultados observados são preocupantes, especialmente em relação a incongruência que inviabiliza a utilização da base de dados. Wyatt \& Wyatt ${ }^{17}$ destacam a necessidade da realização de avaliações ao longo de todo o processo de qualquer sistema de informação. Desde a implantação do Sinan têm sido realizadas sucedidas modificações estruturais para melhorar a adequação da informação disponibilizada, de forma a atender às normas e regulamentos definidos para o Sistema Único de Saúde (SUS), e de prover respostas às demandas da população. Já na migração da base de dados do Sinan DOS para o Sinan Windows foram incluídos procedimentos para identificar as principais inconsistências na base de dados ${ }^{2}$. A complexidade da ficha de notificação de violência, no entanto, requer um aperfeiçoamento das estratégias de críticas na entrada de dados, de forma a proporcionar informações válidas.

A maioria da incongruência observada poderia ser evitada com programação, considerando a orientação do próprio instrutivo de preenchimento da ficha de notificação ${ }^{14}$. O campo lesão autoprovocada deveria ser de digitação obrigatória, o que evitaria a perda de um terço da base de dados, como observado neste estudo. Adicionalmente, deveria ter preenchimento automático com "Não se aplica" para todos os campos referentes à violência interpessoal e no campo tipo de violência, apenas a opção "outros" deveria ficar ativa, para atender a recomendação do instrutivo.

Os erros também poderiam ser minimizados se a categoria "Não se Aplica" fosse incluída nos campos: tipo de violência, ciclo de vida do provável autor da violência, número e sexo dos agressores envolvidos, vínculo de parentesco e violência relacionada ao trabalho; e com preenchimento automático nos casos de tentativa de suicídio e autoagressão. A mesma estratégia poderia ser implantada nos campos escolaridade para vítimas menores de seis anos, situação conjugal para menores de dez anos, trabalho infantil para maiores de quinze anos, pornografia infantil para maiores de dezenove anos, e para os campos restritos ao sexo biológico feminino (gestante, coleta de secreção vaginal, aborto, etc.) em que a vítima é do sexo masculino. No campo identidade de gênero, as categorias "travesti" e "mulher transexual" não deveriam ficar ativas para digitação no caso de notificação de vítima do sexo biológico feminino, e na categoria "homem transexual" para vítima do sexo masculino.

É possível que alguns problemas tenham sido ocasionados por erros de digitação e outros pela própria estruturação dos campos na ficha. Assim, no campo tipo de violência, por exemplo, tem a opção de marcar: "sim", "não" e "ignorado" para cada tipo; o que induz o profissional a registrar mais de um tipo de violência, embora a recomendação seja registrar apenas o tipo principal. No caso de violência psicológica, por exemplo, em que o agressor também utilizou de um instrumento para atingir a vítima apenas a violência física deveria ser registrada; e no caso de estupro a prioridade poderia ser o registro de violência sexual. Não obstante, isto possa ser difícil de definir em alguns casos. Na situação de tráfico de seres humanos com violência sexual, por exemplo, qual seria o tipo de violência principal? Por outro lado, registrar apenas o principal tipo de violência seria a melhor estratégia para conhecer as características deste fenômeno na nossa sociedade?

Ainda são poucos os estudos sobre consistência das bases de dados. Em estudo de revisão dos artigos que avaliaram a qualidade das informações dos SIS $^{18}$ foram analisadas as dimensões: acessibilidade, clareza metodológica, cobertura, completitude, confiabilidade, consistência, não-duplicidade, oportunidade e validade. Dos 78 artigos incluídos no estudo apenas um avaliou consistência ${ }^{19}$, mas não se referia à consistência interna entre os campos do sistema de informação. Este estudo estimou inconsistência na taxa de mortalidade infantil dos estados, capitais e macrorregiões do país; com base na comparação das estimativas oficiais fornecidas pelo IBGE e dados oriundos das bases do sistema de informação de mortalidade (SIM) e de nascidos vivos (Sinasc).

Em levantamento realizado, foram identificados apenas dois estudos que avaliaram consistência, e a propósito nos dados da ficha de notificação de violência ${ }^{20,21}$. Um estudo analisou as notificações de Recife ${ }^{20}$ no período de 2009 a 2012, tendo encontrado uma média de $1 \%$ de inconsistência entre os campos avaliados, sendo que a maior proporção $(10,1 \%)$ ocorreu entre os campos violência psicológica e meio de agressão. $\mathrm{O}$ outro restringiu a avaliação às notificações de violência sexual contra 
a mulher em Santa Catarina ${ }^{21}$ no período de 2008 a 2013, em que a média de inconsistência dos campos analisados foi de 1,1\%. O denominador utilizado em ambos os estudos, no entanto, foi o total de fichas de notificação, ao invés do total de fichas com as categorias dos campos envolvidos na análise, o que pode ter subestimado este indicador. $\mathrm{O}$ total de fichas só deveria ser utilizado como denominador para descrever o percentual de fichas que ficaram comprometidas para análise em função dos erros encontrados. Infelizmente esta informação não está disponível nos estudos supracitados e não pode ser avaliada neste estudo, porque foram utilizados dados agregados.
Os problemas encontrados sinalizam a necessidade de treinamento dos profissionais que atuam no nível local em relação ao instrutivo de preenchimento da ficha de notificação, bem como, dos conceitos que são abordados para propiciar uma classificação adequada. Ademais, os gestores municipais precisam criar estratégias para revisar os dados, identificar as inconsistências e corrigi-las antes de encaminhar os dados para o nível estadual. As informações inadequadas poderão influenciar as políticas de saúde e dificultar o enfrentamento das violências na sociedade.

\section{REFERÊNCIAS}

1. Lima CRA, Leal CD, Dias EP, Gonzalez FL, Santos HL, Silva MEM, et al. Departamento de Informática do SUS - DATASUS: a experiência de disseminação de informações em saúde. In: Ministério da Saúde. A experiência brasileira em sistemas de informação em saúde: produção e disseminação de informações sobre saúde no Brasil. Brasília: Organização Pan-Americana da Saúde, Fundação Oswaldo Cruz; 2009. p. 109-28. (Série B Textos Básicos de Saúde, vol. 1).

2. Souza WV, Domingues CMAS. Notificação compulsória de doenças e agravos no Brasil: um breve histórico sobre a criação do Sistema de Informação de Agravos de Notificação - SINAN. In: Ministério da Saúde. A experiência brasileira em sistemas de informação em saúde: produção e disseminação de informações sobre saúde no Brasil. Brasília: Organização Pan-Americana da Saúde, Fundação Oswaldo Cruz; 2009. p. 39-48. (Série B Textos Básicos de Saúde, vol. 1).

3. Brasil. Secretaria de Vigilância em Saúde. Departamento de Vigilância Epidemiológica. Guia de vigilância epidemiológica. 7. ed. Brasília: Ministério da Saúde; 2009

4. Brasil. Ministério da Saúde. Portaria nº 104, de 25 de janeiro de 2011. Define as terminologias adotadas em legislação nacional, conforme disposto no Regulamento Sanitário Internacional 2005 (RSI 2005), a relação de doenças, agravos e eventos em saúde pública de notificação compulsória em todo o território nacional e estabelecer fluxo, critérios, responsabilidades e atribuições aos profissionais e serviços de saúde. Diário Oficial da União, Brasília, 26 de janeiro de 2011; Seção 1:37.

5. Brasil. Secretaria de Vigilância em Saúde. Coordenação-Geral de Desenvolvimento da Epidemiologia em Serviços. Guia de vigilância em saúde. vol. 3. Brasília: Ministério da Saúde; 2017.

6. Brasil. Ministério da Saúde. Portaria n 1271, de 6 de junho de 2014. Define a lista Nacional de Notificação Compulsória de doenças, agravos e eventos de saúde pública nos serviços de saúde públicos e privados em todo território nacional, nos termos do anexo, e dá outras providências. Diário Oficial da União, Brasília, 9 de junho de 2014; Seção 1:67.

7. Brasil. Secretaria de Vigilância em Saúde. Departamento de Vigilância de Doenças e Agravos Não Transmissíveis e Promoção da Saúde. Instrutivo de notificação de violência interpessoal e autoprovocada. Brasília: Ministério da Saúde; 2014

8. Brasil. Estatuto da criança e do adolescente: lei federal $n^{\circ} 8.069$, de 13 de julho de 1990. Rio de Janeiro: Imprensa Oficial; 2002.

9. Brasil. Estatuto do idoso: lei federal no 10.741 , de 01 de outubro de 2003. Brasília: Secretaria Especial dos Direitos Humanos; 2004.
10. Brasil. Lei federal no 10.778 , de 24 de novembro de 2003. Estabelece a notificação compulsória, no território nacional, do caso de violência contra a mulher que for atendida em serviços de saúde públicos ou privados. Diário Oficial da União, Brasília, 25 de novembro de 2003; Seção 1:11.

11. Brasil. Ministério da Saúde. Portaria no 1876, de 14 de agosto de 2006. Institui Diretrizes Nacionais para Prevenção do Suicídio, a ser implantadas em todas as unidades federadas, respeitadas as competências das três esferas de gestão. Diário Oficial da União, Brasília, 15 de agosto de 2006; Seção 1:65.

12. Paim I, Nehmy RMQ, Guimarães C. Problematização do conceito de "qualidade" da informação. Perspect Cienc Inf. 1996;1:113-22.

13. Campbell SE, Campbell MK, Grimshaw JM, Walker AE. A systematic review of discharge coding accuracy. J Public Health Med. 2001;23(3):205-11. http://dx.doi.org/10.1093/pubmed/23.3.205. PMid:11585193.

14. Brasil. Secretaria de Vigilância em Saúde. Departamento de Vigilância de Doenças e Agravos Não Transmissíveis e Promoção da Saúde. Viva: instrutivo de notificação de violência interpessoal e autoprovocada. 2. ed. Brasília: Ministério da Saúde; 2016.

15. Brasil. Lei federal $n^{\circ} 10.097$, de 19 de agosto de 2000. Altera dispositivos da Consolidação das Leis do Trabalho - CLT, aprovada pelo Decreto-Lei no 5.452, de 1o de maio de 1943. Diário Oficial da União, Brasília, 20 de dezembro de 2000; Seção 1:2.

16. Brasil. Lei federal $n^{\circ} 12.015$, de 7 de agosto de 2009. Altera o Título VI da Parte Especial do Decreto-Lei no 2.848, de 7 de dezembro de 1940 - Código Penal, e o art. $1^{\circ}$ da Lei no 8.072, de 25 de julho de 1990, que dispõe sobre os crimes hediondos, nos termos do inciso XLIII do art. $5^{\circ}$. da Constituição Federal e revoga a Lei no 2.252, de $1^{\circ}$. De julho de 1954, que trata de corrupção de menores. Diário Oficial da União, Brasília, 10 de agosto de 2009; Seção 1:2.

17. Wyatt JC, Wyatt SM. When and how to evaluate health information systems? Int J Med Inform. 2003;69(2-3):251-9. http://dx.doi.org/10.1016/ S1386-5056(02)00108-9. PMid:12810128.

18. Lima CR, Schramm JM, Coeli CM, Silva ME. Revisão das dimensões de qualidade dos dados e métodos aplicados na avaliação dos sistemas de informação em saúde. Cad Saude Publica. 2009;25(10):2095-109. http:// dx.doi.org/10.1590/S0102-311X2009001000002. PMid:19851611.

19. Szwarcwald CL, Leal MC, Andrade CL, Souza PR Jr. Estimação da mortalidade infantil no Brasil: o que dizem as informações sobre óbitos e nascimentos do Ministério da Saúde? Cad Saude Publica. 2002;18(6):1725-36. http:// dx.doi.org/10.1590/S0102-311X2002000600027. PMid:12488900. 
20. Abath MB, Lima MLLT, Lima OS, Silva MCM, Lima MLC. Avaliação da completitude, da consistência e da duplicidade de registros de violências do Sinan em Recife, Pernambuco, 2009-2012. Epidemiol Serv Saude. 2014;23(1):131-42. http://dx.doi.org/10.5123/S1679-49742014000100013.

21. Delziovo CR, Bolsoni CC, Lindner SR, Coelho EBS. Qualidade dos registros de violência sexual contra a mulher no Sistema de Informação de Agravos de Notificação (Sinan) em Santa Catarina, 2008-2013. Epidemiol Serv Saude. 2018;27(1):e20171493. http://dx.doi.org/10.5123/S1679-49742018000100003. PMid:29412348.

Recebido em: Abr. 05, 2018

Aprovado em: Ago. 01, 2018 